\title{
Electrostatic charging of trigger actuated spray devices
}

\author{
L.F. Gaunt and J.F. Hughes.
}

Bioelectrostatics Research Centre, Department of Electronics and Computer Science, University of Southampton, Highfield, Southampton, UK, SO17 1BJ

\begin{abstract}
Electrostatic charging has long been used to improve the efficiency of a range of sprayed liquids. However, the benefits have not until recently been available for exploitation by domestic sprays due to the need for a high voltage power supply. A minimum charge-to-mass ratio $(\mathrm{q} / \mathrm{m})$ of $1 \times 10^{-4} \mathrm{C} / \mathrm{kg}$ is generally considered necessary to elicit electrostatic benefits. This level of charge can now be imparted to liquids atomised from trigger-actuated spray devices by a passive system, requiring no power supply. Induction charging was achieved using a triboelectrically charged aluminium electrode. The $\mathrm{q} / \mathrm{m}$ of the sprayed liquid was dependent upon the charge residing on the induction electrode. The induction electrode was electrically isolated and required a charge of between 0.7 and $1.3 \times 10^{-8} \mathrm{C}$ to deliver a water spray with a $\mathrm{q} / \mathrm{m}$ of $1 \times 10^{-4} \mathrm{C} / \mathrm{kg}$. This level of static charge was readily attained by tribocharging the aluminium with polythene. Once generated, sufficient charge remained on the electrode surface to charge successive sprays without the need for regeneration. The performance advantages for a spray charged in this manner include attraction to and targeting of surfaces and wrap-around onto surfaces not in the direct line of sight.
\end{abstract}

\section{Introduction}

Electrostatic charging is a familiar method used for improving the efficiency of a range of sprayed liquids, for example in electrostatic crop spraying and painting applications. The charge is usually imparted either by induction or corona methods, using a high voltage power supply, or by tribocharging in powder coating systems. Typically a minimum charge-to-mass ratio $(\mathrm{q} / \mathrm{m})$ of $1 \times 10^{-4} \mathrm{C} / \mathrm{kg}$ is considered necessary to elicit electrostatic benefits [1;2]. Domestic sprays, such as the familiar hand-held, pressure-pack (PP) device have not previously exploited the advantages of electrostatic spray technology, primarily due to the necessity for a power supply that adds prohibitive cost and complexity to a simple domestic device. Recent research, however, has led to the development of a domestic pressure-pack dispenser that produces an aerosol of droplets that carry this level of charge. Natural charge exchange processes that occur during atomisation have been enhanced to attain the elevated $\mathrm{q} / \mathrm{m}$ values [3]. This has been achieved through the optimisation of formulation and pressure parameters combined with an actuator incorporating a novel orifice design, which promotes shearing of the electrical double layer at the liquid/solid interface. This has led to the development of a domestic insecticide spray with significantly improved insect targeting characteristics, leading to substantial improvements in insect knockdown and mortality [2;4].

Another form of spray device that is commonly encountered in the domestic environment is the trigger actuated spray. This is used to dispense a variety of water-based detergents and cleaning products such as furniture polish, kitchen and bathroom cleaners and disinfectants. As the majority of these applications involve targeting a surface, electrostatic spraying would be beneficial in enhancing product deposition and delivering a more even coating. A threshold charge-to-mass ratio value of $1 \times 10^{-4} \mathrm{C} / \mathrm{kg}$ would be 
predicted as a minimum to achieve these advantages, based on previous research findings $[1 ; 2]$. In attempting to achieve this level of charge, promoting the shearing of the electrical double layer (as with PP sprays) was found not to be successful. This was believed to be because of the difference in atomisation characteristics and due to the widespread use of aqueous formulations. Therefore, an alternative way of producing a charge-on-demand spray was required. One solution was an induction charging system using an electrically isolated electrode at high potential, designed such that atomisation of the spray occurred under the influence of a high electric field. The liquid reservoir was grounded and acted as a counter electrode. Charged droplets were formed as they broke away from the reservoir. The charge on the induction electrode could be established by triboelectric charging, which could occur during the action of squeezing the actuation trigger.

Presented here are preliminary investigations into the level of charge that is required on such an induction electrode to produce a spray with a $\mathrm{q} / \mathrm{m}$ above the threshold value of $1 \times 10^{-4} \mathrm{C} / \mathrm{kg}$, and the $\mathrm{q} / \mathrm{m}$ of successive sprays.

\section{Materials and Methods}

\subsection{Design of the charge-on-demand trigger spray device}

A trigger spray device was designed in order to test the concept of using an induction electrode to charge a liquid as it was atomised from a conventional trigger pack. A commercially available trigger pack was modified by covering the outer plastic shroud with aluminium foil as shown in Figure 1. The foil was extended to the front of the shroud to create an induction zone around the spray orifice. The distance across this gap was $8.0 \mathrm{~mm}$, and the spray orifice was positioned central within this. The foil was positioned so that it remained electrically isolated from the other parts of the trigger device and the user during actuation. For some experiments, static charge was deposited on the induction electrode by contact with a voltage source (Brandenburg Alpha III), typically using a voltage of between 1 and $8 \mathrm{kV}$ DC. The polarity of charge carried by the droplets was always opposite to that of the induction electrode. The liquid reservoir was grounded via a wire from the inside of the container and running over the lip to the outside. The induction electrode was also charged by rubbing with a piece of polyethylene sheet. The charge typically achieved on the electrode in this way was between 1 and $3 \times 10^{-8} \mathrm{C}$, the polarity always being positive.

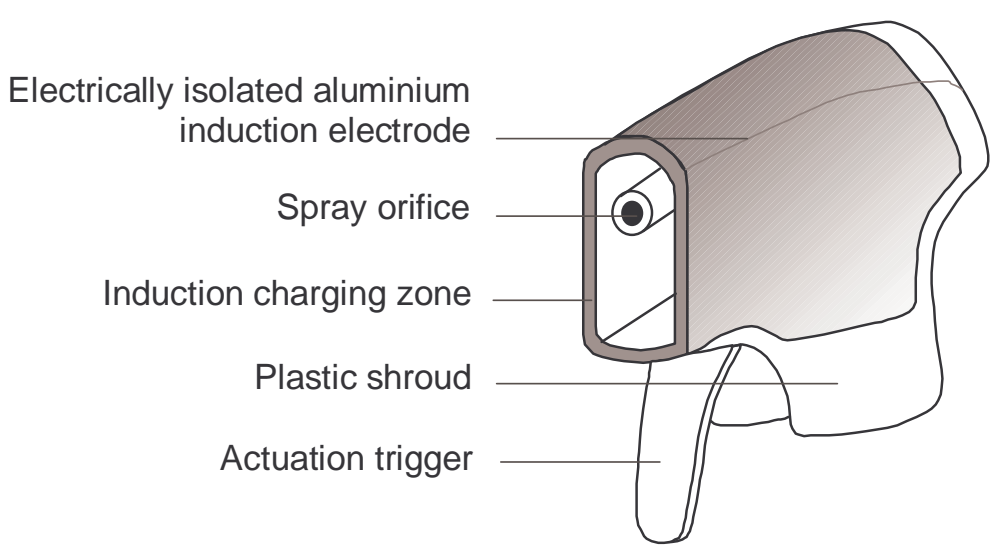

Figure 1 Schematic drawing of the charge-on-demand trigger spray device 


\subsection{Measuring charge and charge-to-mass ratio}

The charge residing on the induction electrode was measured by lowering the trigger assembly into a Faraday pail connected to an electrometer (Keithley Instruments, 610C). Similarly, the charge on the spray from the trigger device was measured by spraying into a Faraday pail with an aperture wide enough to allow the spray plume to enter. The mean $\mathrm{q} / \mathrm{m}$ of the spray was calculated, based on a minimum of 5 replicates.

\subsection{Influence of induction electrode potential on spray $q / m$}

The electrical potential of the induction electrode was expected to determine the level of charge achieved on the liquid during atomisation, as it would determine the intensity of the electric field. The electrical potential of the induction electrode was controlled by contacting the electrode with a range of voltages between 1 and $8 \mathrm{kV} \mathrm{DC}$. The electrode polarity was positive, so the spray polarity was negative. The $\mathrm{q} / \mathrm{m}$ of the liquid was measured, and the potential restored before another measurement was taken. The test liquids were tap water and a 5\% detergent solution. Statistical analysis (t-test) was performed to identify the significance in differences between the $\mathrm{q} / \mathrm{m}$ of the two test liquids.

\section{4. $Q / m$ of successive charges}

As charging the sprayed liquids by induction was not expected to remove the charge from the induction electrode, successive trigger actuations should be charged. However, the charge on the induction electrode might be expected to decrease by natural leakage, resulting in a corresponding decrease in the $\mathrm{q} / \mathrm{m}$ of the spray. To investigate this effect, the $\mathrm{q} / \mathrm{m}$ of tap water was measured for the first, second and third successive trigger actuations. The charge on the induction electrode was between 1 and $2 \times 10^{-8} \mathrm{C}$, achieved by triboelectric charging with polyethylene sheet.

\section{Results and Discussion}

\subsection{Influence of induction electrode potential on spray $q / m$}

The results in Figure 2 show the relationship between the charge on the induction electrode and the $\mathrm{q} / \mathrm{m}$ of the resultant spray for tap water and a $5 \%$ detergent solution. As the charge on the electrode was increased, so the $\mathrm{q} / \mathrm{m}$ of the sprays increased. This was seen for both liquids, although the $q / m$ of the water was significantly higher $(p<0.05)$ than that of the detergent for the same level of charge on the induction electrode. The increase in $\mathrm{q} / \mathrm{m}$ as the level of charge on the induction electrode increased would have occurred as a result of the intensification of the electric field at the point of liquid atomisation. Thus, a higher charge was induced in the forming droplets as they broke away from the grounded liquid reservoir.

The difference in $\mathrm{q} / \mathrm{m}$ between the tap water and detergent solution could have arisen through differences in conductivity, viscosity or surface tension; factors that can effect the charging or atomisation characteristics.

The natural $\mathrm{q} / \mathrm{m}$ of these liquids without a charge on the induction electrode is also shown in Fig. 2. The values are low, in the region of $7 \times 10^{-6} \mathrm{C} / \mathrm{kg}$ for water. The charge is believed to arise from shearing of the electrical double layer that exists at the liquid-solid interface. 


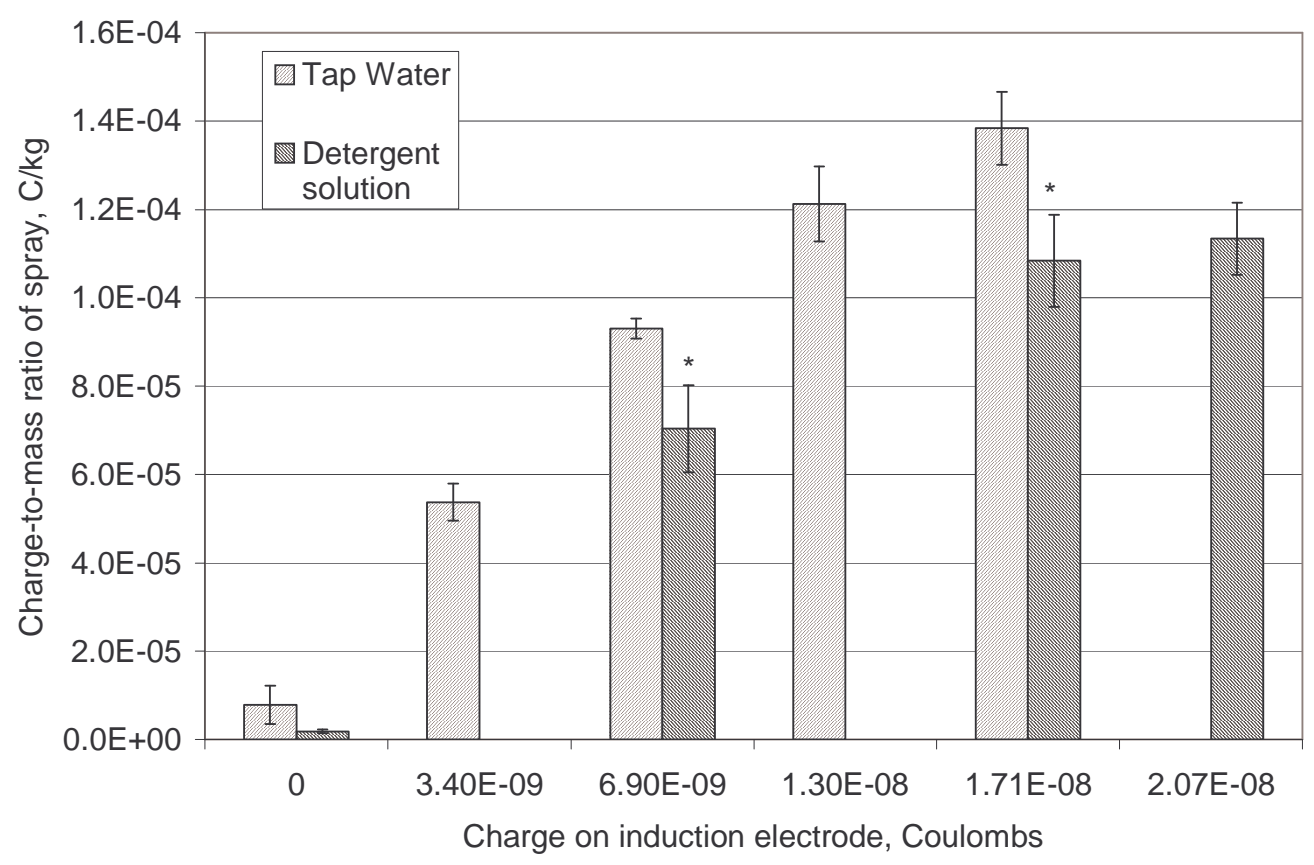

Figure 2 Relationship between the charge on the induction electrode and the charge-to-mass ratio of water and detergent sprays. Based on 5 replicates, error bars indicate the standard error of the mean, ${ }^{*}$ indicates statistically significant difference between $\mathrm{q} / \mathrm{m}$ values $(\mathrm{p}<0.05)$.

\section{2. $Q / m$ of successive charges}

Figure 3 shows that charge is induced in successive sprays from the trigger, but that the $\mathrm{q} / \mathrm{m}$ of the sprays decreased. This is probably due to the charge carried on the induction electrode decreasing with time or with successive sprays. The charge on the induction electrode could be expected to decrease over time due to charge leakage from the surface and neutralisation. It is not necessarily lost as an outcome of the sprays. The results show that triboelectric charging of the induction electrode is a suitable method for charging the spray to the theoretical threshold of $1 \times 10^{-4} \mathrm{C} / \mathrm{kg}$. The natural $\mathrm{q} / \mathrm{m}$ of the sprays without the induction electrode being charged is much lower, but also shows a decrease with successive actuations. The natural $\mathrm{q} / \mathrm{m}$ may decrease with successive actuations due to the changes in the electrochemical characteristics of the interface following each actuation. 


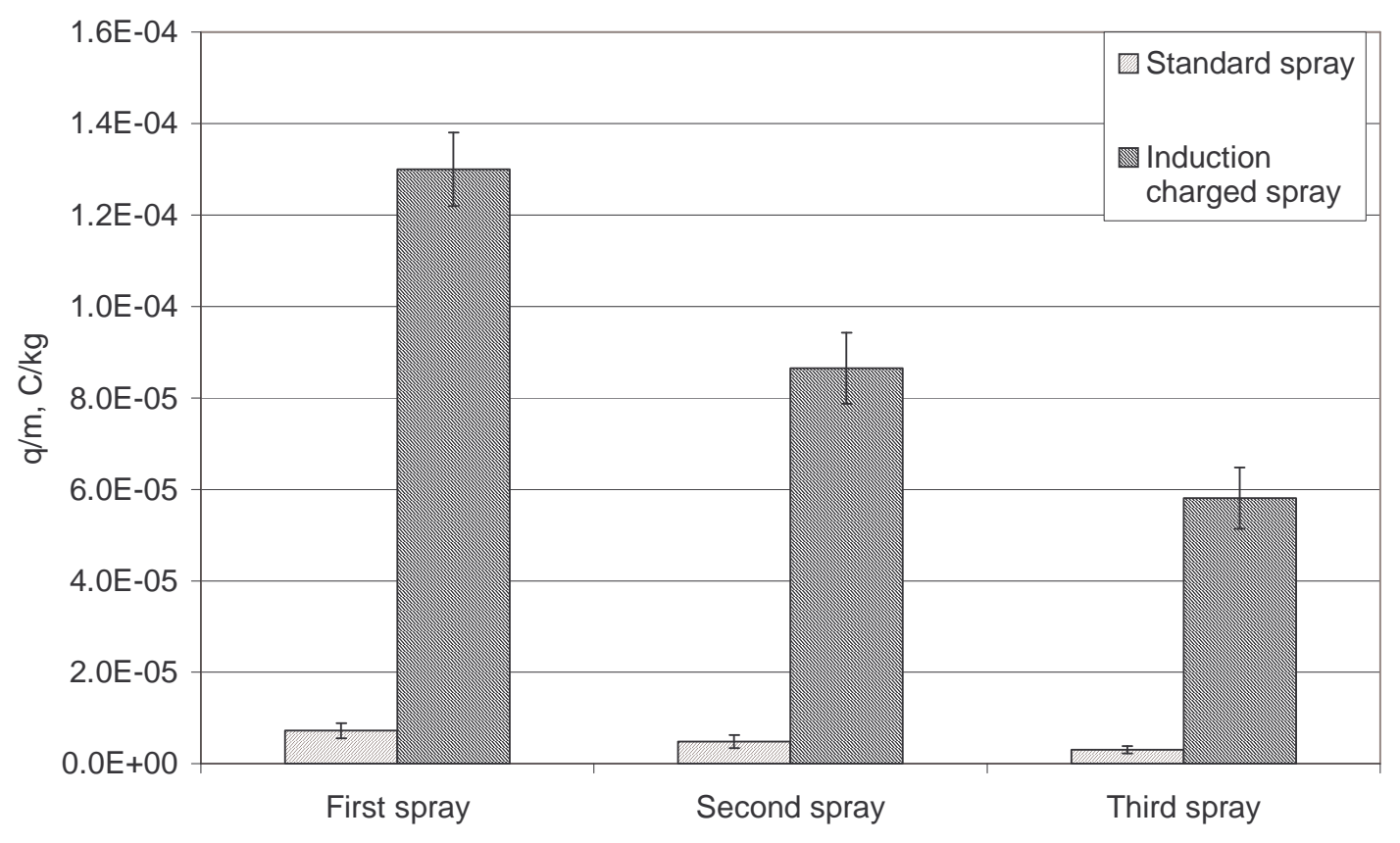

Figure 3 Charge-to-mass ratio of successive trigger sprays of tap water. Tribocharged induction electrode charge of between 1 and $2 \times 10^{-8} \mathrm{C}$. Based on 5 replicates, error bars show the standard error of the mean.

\section{Conclusions}

It has been demonstrated that it is possible to charge sprays produced by trigger actuated devices by a passive system, not requiring a voltage source. Charge-to-mass ratio values in excess of $1 \times 10^{-4} \mathrm{C} / \mathrm{kg}$ have been demonstrated on water and detergent solutions. This has been achieved by atomising the liquid in an electric field created by an electrically isolated induction electrode at high potential. The minimum charge on the induction electrode for tap water was between 0.7 and $1.3 \times 10^{-8} \mathrm{C}$, while for a $5 \%$ detergent solution it was between 0.7 and $1.7 \times 10^{-8} \mathrm{C}$. In these investigations the charge was imparted to the electrode by contact with a voltage source for reproducibility. Tribocharging of the aluminium against polyethylene sheet consistently achieved charges of between 1 and $3 \times$ $10^{-8} \mathrm{C}$. This was shown to achieve water sprays of in excess of $1.2 \times 10^{-4} \mathrm{C} / \mathrm{kg}$. It is envisaged that in the final device the triboelectric charging will be generated during the process of trigger actuation [5]. Work is ongoing to design this contact process into existing trigger devices, and to optimise the geometry of the charge induction zone.

The electrical potential of the induction electrode influenced the charge-to-mass ratio of the sprayed liquid. As the electrical potential of the induction electrode was increased, the $\mathrm{q} / \mathrm{m}$ of the liquid spray was increased. This was observed for tap water and a detergent solution, although the $\mathrm{q} / \mathrm{m}$ of these differed for the same electrode potential. Factors such as surface tension, conductivity and viscosity will also have considerable influence on the charge characteristics, through their effect on the atomisation process and the time rate of charge transfer [6;7].

The benefits of a charge-on-demand trigger actuated spray device reflect those already know to industrial applications of electrostatic spraying. These include attraction to 
the target surface, wrap-around onto surfaces not in the direct line of sight and more even coverage resulting from space charge effects and electrostatic attraction. As a result, the efficacy and performance of common domestic sprays could be substantially improved.

\section{Acknowledgements}

This work was funded by Reckitt Benckiser Ltd (UK), whose support is gratefully acknowledged.

\section{References}

[1] Singh S, O'Neil BC, Bright SW. 1978 Journal of Electrostatics 4:325-334.

[2] Gaunt LF, Hughes JF, Harrison N. 2003 Journal of Electrostatics 57:35-47.

[3] Fox R, Harrison N, Hughes JF, Whitmore LF. 1999 patent WO 99/01227.

[4] Whitmore LF, Hughes JF, Harrison N, Abela M, O'Rourke P. 2001 Pest Management Science 57 (5) :432-436.

[5] Hughes JF, Gaunt LF. Spraying devices. 2001 Patent application GB 0116543.0.

[6] Cross J. Electrostatics: principles, problems and applications. 1987 Adam Hilger, Bristol.

[7] Law SE. Electrostatic atomization and spraying. In: Chang J, Kelly A.J., Crowley J.M., editors. 1995 Handbook of electrostatic processes. New York: Dekker, pp 413-440. 